\title{
Hardy-Type Space Associated with an Infinite-Dimensional Unitary Matrix Group
}

\author{
Oleh Lopushansky \\ Institute of Mathematics, University of Rzeszów, 16A Rejtana Street, 35-310 Rzeszów, Poland \\ Correspondence should be addressed to Oleh Lopushansky; ovlopusz@univ.rzeszow.pl
}

Received 23 March 2013; Accepted 18 June 2013

Academic Editor: Qing-Wen Wang

Copyright (C) 2013 Oleh Lopushansky. This is an open access article distributed under the Creative Commons Attribution License, which permits unrestricted use, distribution, and reproduction in any medium, provided the original work is properly cited.

\begin{abstract}
We investigate an orthogonal system of the homogenous Hilbert-Schmidt polynomials with respect to a probability measure which is invariant under the right action of an infinite-dimensional unitary matrix group. With the help of this system, a corresponding Hardy-type space of square-integrable complex functions is described. An antilinear isomorphism between the Hardy-type space and an associated symmetric Fock space is established.
\end{abstract}

\section{Introduction}

We investigate an orthogonal system of the Hilbert-Schmidt polynomials in the space $L_{\chi}^{2}$ of square-integrable complex functions on the projective limit $\mathfrak{U}=\lim U(m)$ of unitary $(m \times m)$-dimensional matrix groups $U(m)(m \in \mathbb{N})$, called the space of virtual unitary matrices and endowed with the projective limit measure $\chi=\lim \chi_{m}$ of the probability Haar measures $\chi_{m}$ on $U(m)$. The measure $\chi$ on the space $\mathfrak{U}$ is invariant under the right action of the infinite-dimensional unitary group $U(\infty) \times U(\infty)$, where $U(\infty)=\bigcup_{m} U(m)$.

The space of virtual unitary matrices $\mathcal{U}$ was studied by Neretin [1] and Olshanski [2]. This notion relates to D. Pickrell's space of virtual Grassmannian [3] and to Kerov, Olshanski, and Vershik's space of virtual permutations [4]. Various spaces of integrable functions with respect to measures that are invariant under infinite-dimensional groups have been widely applied in stochastic processes [5], infinitedimensional probability $[6,7]$, complex analysis $[8]$, and so forth.

The main results of the present paper are Theorems 67 that describe a Hardy-type subspace $\mathscr{H}_{\chi}^{2} \subset L_{\chi}^{2}$ spanned by the finite type homogenous Hilbert-Schmidt polynomials that are generated by an associated symmetric Fock space.

\section{Preliminaries}

We consider the following infinite-dimensional unitary matrix groups:

$$
\begin{gathered}
U(\infty)=\bigcup\{U(m): m \in \mathbb{N}\}, \\
U^{2}(\infty):=U(\infty) U(\infty),
\end{gathered}
$$

where $U(m)$ is the group of unitary $(m \times m)$-matrices which is identified with the subgroup in $U(m+1)$ fixing the $(m+$ $1)$ th basis vector. In other words, $U(\infty)$ is the group of infinite unitary matrices $u=\left[u_{i j}\right]_{i, j \in \mathbb{N}}$ with finitely many matrix entries $u_{i j}$ distinct from $\delta_{i j}$. We equip every group $U(m)$ with the probability Haar measure $\chi_{m}$.

Following [1,2], every matrix $u_{m} \in U(m)$ with $m>1$, we write in the following block matrix form:

$$
u_{m}=\left[\begin{array}{cc}
z_{m-1} & a \\
b & t
\end{array}\right] \text {, }
$$

corresponding to the partition $m=(m-1)+1$ so that $z_{m-1} \in$ $U(m-1)$ and $t \in \mathbb{C}$. Over the group $U(\infty)$ (resp., $U(m))$ the right action is well defined:

$$
u \cdot g=w^{-1} u v
$$


where $u$ belongs to $U(\infty)$ (resp., to $U(m))$ and $g=(v, w)$ belongs to $U^{2}(\infty)$ (resp., to $\left.U^{2}(m):=U(m) \times U(m)\right)$. In [1, Proposition 0.1], [2, Lemma 3.1], it was proven that the following Livšic-type mapping:

$$
\pi_{m-1}^{m}: U(m) \ni u_{m} \longrightarrow u_{m-1} \in U(m-1),
$$

such that

$$
\left[\begin{array}{cc}
z_{m-1} & a \\
b & t
\end{array}\right] \longmapsto \begin{cases}z_{m-1}-a(1+t)^{-1} b: & t \neq-1 \\
z_{m-1}: & t=-1\end{cases}
$$

(which is not a group homomorphism) is Borel and surjective onto $U(m-1)$ and commutes with the right action of $U^{2}(m-$ 1).

As is known [1, Theorem 1.6], the pullback of the probability Haar measure $\chi_{m-1}$ on $U(m-1)$ under the mapping $\pi_{m-1}^{m}$ is the probability Haar measure $\chi_{m}$ on $U(m)$, that is,

$$
\chi_{m-1} \circ \pi_{m-1}^{m}=\chi_{m} .
$$

Let $U^{\prime}(m) \subset U(m)$ be the subset of unitary matrices which do not have $\{-1\}$, as an eigenvalue. Then, $U^{\prime}(m)$ is open in $U(m)$, and the complement $U(m) \backslash U^{\prime}(m)$ is a $\chi_{m^{-}}$ negligible set. Moreover (see [2, Lemma 3.11]), the mapping

$$
\pi_{m-1}^{m}: U^{\prime}(m) \longrightarrow U^{\prime}(m-1)
$$

is continuous and surjective.

Consider the projective limits, taken with respect to the surjective Borel projections $\pi_{m-1}^{m}$ and their continuous restrictions $\left.\pi_{m-1}^{m}\right|_{U^{\prime}(m)}$, respectively,

$$
\mathfrak{U}=\lim _{\leftarrow} U(m), \quad \mathfrak{U}^{\prime}=\lim _{\leftarrow} U^{\prime}(m),
$$

called the spaces of virtual unitary matrices. Notice that $\mathcal{U}$ is a Borel subset in the Cartesian product $X_{m \in \mathbb{N}} U(m)=\{u=$ $\left.\left(u_{m}\right): u_{m} \in U(m)\right\}$ endowed with the product topology, because all mapping $\pi_{m-1}^{m}$ are Borel. Moreover, the canonical projections

$$
\pi_{m}: \mathfrak{U} \longrightarrow U(m), \quad \pi_{m}: \mathfrak{U}^{\prime} \longrightarrow U^{\prime}(m),
$$

such that $\pi_{m-1}=\pi_{m-1}^{m} \circ \pi_{m}$, are surjective by surjectivity of $\pi_{m-1}^{m}$ and $\left.\pi_{m-1}^{m}\right|_{U^{\prime}(m)}$.

Following [2, Lemma 4.8], [1, Section 3.1], with the help of the Kolmogorov consistent theorem, we uniquely define a probability measure $\chi$ on $\mathfrak{U}^{\prime}$ as the projective limit under the mapping (6),

$$
\chi=\lim _{\leftarrow} \chi_{m}
$$

which satisfies the equality $\chi=\chi_{m} \circ \pi_{m}$ for all $m \in \mathbb{N}$. On $\mathfrak{U} \backslash$ $\mathfrak{U}^{\prime}$, the measure $\chi$ is zero, because $\chi_{m}$ is zero on $U(m) \backslash$ $U^{\prime}(m)$ for all $m \in \mathbb{N}$.

Using (3), right action of the group $U^{2}(\infty)$ on the space of virtual unitary matrices $\mathcal{U}$ can be defined (see [2, Definition 4.5]) as follows:

$$
\pi_{m}(u \cdot g)=w^{-1} \pi_{m}(u) v, \quad u \in \mathfrak{U},
$$

where $m$ is so large that $g=(v, w) \in U^{2}(m)$.
The canonical dense embedding $\imath: U(\infty) \rightarrow \mathfrak{U}$ to any element $u_{m} \in U(m)$ assigns the unique sequence $u=\left(u_{l}\right)_{l \in \mathbb{N}}$, such that

$$
\begin{gathered}
l: U(m) \ni u_{m} \longmapsto\left(u_{l}\right) \in \mathcal{U}, \\
u_{l}= \begin{cases}\pi_{l}^{l+1} \circ \ldots \circ \pi_{m-1}^{m}\left(u_{m}\right): & l<m, \\
u_{m}: & l=m, \\
{\left[\begin{array}{cc}
u_{m} & 0 \\
0 & \mathbb{1}_{l-m}
\end{array}\right]:} & l>m,\end{cases}
\end{gathered}
$$

where $\mathbb{1}_{l-m}$ is the unit in $U(l-m)$. So, the image $\imath$ 。 $U(\infty)$ consists of stabilizing sequences in $\mathcal{U}$ (see [2, Section 4]).

\section{Invariant Probability Measure}

In what follows, we will endow the space of virtual unitary matrices $\mathfrak{U}$ with the measure $\chi=\lim \chi_{m}$. A complex function on $\mathfrak{U}$ is called cylindrical [2, Definition 4.5] if it has the following form:

$$
f(u)=\left(f_{m} \circ \pi_{m}\right)(u), \quad u \in \mathfrak{U},
$$

for a certain $m \in \mathbb{N}$ and a certain complex function $f_{m}$ on $U(m)$.

Any continuous bounded function $f$ on $\mathfrak{U}^{\prime}$ has a unique $\chi$-essentially bounded extension on $\mathfrak{U}$, because the set $\mathfrak{U} \backslash \mathfrak{U}^{\prime}$ is $\chi$-negligible. Therefore, if the function $U^{\prime}(m) \ni \pi_{m}(u) \mapsto$ $f_{m}\left[\pi_{m}(u)\right]$ in the definition (13) is continuous and bounded, then the corresponding cylindrical function $f$ is $\chi$ essentially bounded.

By $\mathscr{L}_{\chi}^{\infty}$, we denote closure of the algebraic hull of all cylindrical $\chi$-essentially bounded functions (13) with respect to the following norm:

$$
\|f\|_{\mathscr{L}_{\chi}^{\infty}}=\underset{u \in \mathfrak{U}}{\operatorname{ess} \sup }|f(u)| .
$$

Lemma 1. The measure $\chi=\lim _{\leftarrow} \chi_{m}$ on $\mathfrak{U}$ is a Radon probability measure such that

$$
\int_{\mathfrak{U}} f(u \cdot g) d \chi(u)=\int_{\mathfrak{U}} f(u) d \chi(u),
$$

for all $g \in U^{2}(\infty)$ and $f \in \mathscr{L}_{\chi}^{\infty}$. For any compact set $K \subset$ $U(m)$ the following equality holds:

$$
(\chi \circ \imath)(K)=\chi_{m}(K) .
$$

Proof. Recall the Prohorov criterion, which is adapted to our notation (see [9, Chapter IX.4.2, Theorem 1] or [6, Theorem 6]): there exists a Radon probability measure $\chi^{\prime}$ on $\mathfrak{U}^{\prime}$ such that

$$
\chi^{\prime}=\left.\chi_{m} \circ \pi_{m}\right|_{\mathfrak{u}^{\prime}} \quad \forall m \in \mathbb{N},
$$

if and only if for every $\varepsilon>0$ there exists a compact set $\mathscr{K}$ in $\mathfrak{U}^{\prime}$ such that the following inequality

$$
\left(\chi_{m} \circ \pi_{m}\right)(\mathscr{K}) \geq 1-\varepsilon \quad \forall m \in \mathbb{N}
$$


holds; in this case, $\chi^{\prime}$ is uniquely determined by means of the formula $\chi^{\prime}(\mathscr{K})=\inf _{m \in \mathbb{N}}\left(\chi_{m} \circ \pi_{m}\right)(\mathscr{K})$, where $\mathscr{K}$ is a compact set in $\mathfrak{U}^{\prime}$.

Let $K_{n} \subset U^{\prime}(n)$ be a compact set with a fixed $n$. Putting $K_{n-1}=\pi_{n-1}^{n}\left(K_{n}\right)$, we have

$$
\chi_{n-1}\left(K_{n-1}\right)=\left(\chi_{n-1} \circ \pi_{n-1}^{n}\right)\left(K_{n}\right)=\chi_{n}\left(K_{n}\right) .
$$

On the other hand, if we put $K_{n+1}=\left[\begin{array}{cc}K_{n} & 0 \\ 0 & 1\end{array}\right]$, then via (6),

$$
\begin{aligned}
\chi_{n+1}\left(K_{n+1}\right) & =\left(\chi_{n} \circ \pi_{n}^{n+1}\right)\left(K_{n+1}\right) \\
& =\left(\chi_{n} \circ \pi_{n}^{n+1}\right)\left[\begin{array}{cc}
K_{n} & 0 \\
0 & 1
\end{array}\right]=\chi_{n}\left(K_{n}\right) .
\end{aligned}
$$

As a consequence, the compact set $\mathscr{K}=\left(K_{m}\right)$ in $\mathfrak{U}^{\prime}$, generated by a compact set $K_{n} \subset U^{\prime}(n)$ with the help of mappings $\pi_{n-1}^{n}$, satisfies the following condition:

$$
\chi_{n}\left(K_{n}\right)=\chi_{m}\left(K_{m}\right) \quad \forall m \in \mathbb{N} \text {. }
$$

The probability Haar measure $\chi_{n}$ is regular on $U(n)$, and the complement $U(n) \backslash U^{\prime}(n)$ is a negligible set. Hence, if $K_{n}$ runs over all compact sets in $U^{\prime}(n)$, then

$$
\sup _{K_{n} \subset U^{\prime}(n)} \chi_{n}\left(K_{n}\right)=1
$$

Therefore, for every $\varepsilon>0$ there exists a compact set $K_{n} \subset$ $U^{\prime}(n)$ such that $\chi_{n}\left(K_{n}\right) \geq 1-\varepsilon$. From (21), it follows that for every $\varepsilon>0$ the compact set $\mathscr{K}$ satisfies the hypothesis of Prohorov's criterion:

$$
\left(\chi_{m} \circ \pi_{m}\right)(\mathscr{K})=\chi_{m}\left(K_{m}\right) \geq 1-\varepsilon \quad \forall m \in \mathbb{N} .
$$

So, in view of this criterion, there exists a unique Radon probability measure $\chi^{\prime}$ on $\mathfrak{U}^{\prime}$ which satisfies the condition (17). However, on the projective limits $\mathfrak{U}^{\prime}=\lim U^{\prime}(m)$, there exists a unique $U^{2}(\infty)$-invariant Radon measure $\chi$, determined by the equality (15). Using the uniqueness property of projective limits, we obtain $\chi^{\prime}=\chi$. The measure $\chi$ on $\mathfrak{U} \backslash \mathfrak{U}^{\prime}$ is defined to be zero, because $\chi_{m}$ is zero on $U(m) \backslash U^{\prime}(m)$.

As a consequence of (21), we obtain (16), because

$$
\chi(\mathscr{K})=\inf _{m \in \mathbb{N}} \chi_{m}\left(K_{m}\right)=\chi_{n}\left(K_{n}\right) .
$$

As is known [1, Proposition 3.2], the measure $\chi$ is $U^{2}(\infty)$ invariant under the right actions (11) on the space $\mathcal{U}$. Hence, for every $f \in \mathscr{L}_{\chi}^{\infty}$, the equality (15) holds.

\section{Shift Groups}

Consider that in the space $\mathscr{L}_{\chi}^{\infty}$, the group of shifts

$$
Q_{g} f(u)=f(u \cdot g), \quad g \in U^{2}(\infty) u \in \mathfrak{U},
$$

is generated by the right action of $U^{2}(\infty)$ over $\mathfrak{U}$. Choosing instead of $U(\infty)$ a compact subgroup $U(m)$ or the compact subgroups

$$
\begin{gathered}
U_{0}=\left\{g_{0}(\vartheta)=\exp (\mathfrak{i} \vartheta): \vartheta \in(-\pi, \pi]\right\}, \\
U_{j}(m)=\left\{g_{m j}(\vartheta)=\mathbb{1}_{j-1} \otimes \exp (\mathfrak{i} \vartheta) \otimes \mathbb{1}_{m-j}: \vartheta \in(-\pi, \pi]\right\} \\
j=1, \ldots, m,
\end{gathered}
$$

we obtain the corresponding subgroups of shifts $Q_{g}$ with elements $g \in U^{2}(m)$ or with elements $g_{0}(\vartheta) \in U_{0}^{2}$ and $g_{m j}(\vartheta) \epsilon$ $U_{j}^{2}(m)$, respectively. Here, $\mathbb{1}_{m}$ means the unit element in $U(m)$.

Lemma 2. For any $f \in \mathscr{L}_{\chi}^{\infty}$ the following equalities:

$$
\begin{gathered}
\int_{\mathfrak{U}} f d \chi=\int_{\mathfrak{U}} d \chi(u) \int_{U^{2}(m)} Q_{g} f(u) d\left(\chi_{m} \otimes \chi_{m}\right)(g), \\
\int_{\mathfrak{U}} f d \chi=\frac{1}{2 \pi} \int_{\mathfrak{U}} d \chi(u) \int_{-\pi}^{\pi} Q_{g(\vartheta)} f(u) d \vartheta,
\end{gathered}
$$

with $g(\vartheta) \in U_{0}^{2}$ or $U_{j}^{2}(m)$ hold.

Proof. For any $f \in \mathscr{L}_{\chi}^{\infty}$, the function $(u, g) \mapsto Q_{g} f(u)=$ $f(u \cdot g)$ is integrable on the Cartesian product $\mathfrak{U} \times U^{2}(m)$. By the Fubini theorem, we obtain

$$
\begin{aligned}
\int_{\mathfrak{U}} d \chi(u) \int_{U^{2}(m)} Q_{g} f(u) d\left(\chi_{m} \otimes \chi_{m}\right)(g) \\
=\int_{U^{2}(m)} d\left(\chi_{m} \otimes \chi_{m}\right)(g) \int_{\mathfrak{U}} Q_{g} f(u) d \chi(u) .
\end{aligned}
$$

This equality yields the required formula (27), because the internal integral on the right-hand side is independent of $g$ and $\int_{U^{2}(m)} d\left(\chi_{m} \otimes \chi_{m}\right)=1$. In turn, putting instead of $U(m)$ the subgroups $U_{0}$ and $U_{j}(m)$, we obtain equalities (28).

\section{The Homogeneous Hilbert-Schmidt Polynomials}

Consider the countable orthogonal Hilbertian sum

$$
\mathrm{E}:=\bigoplus_{m \in \mathbb{N}} \mathbb{C}^{m}=\left\{x=\left(x_{m}\right): x_{m} \in \mathbb{C}^{m},\|x\|_{\mathrm{E}}<\infty\right\},
$$

with the scalar product $\langle x \mid y\rangle_{\mathrm{E}}=\sum_{m}\left\langle x_{m} \mid y_{m}\right\rangle_{\mathbb{C}^{m}}$, where every coordinate $x_{m} \in \mathbb{C}^{m}$ is identified with its image $(0, \ldots$, $\left.0, x_{m}, 0, \ldots\right) \in \mathrm{E}$ under the embedding $\mathbb{C}^{m} \rightarrow \mathrm{E}$.

Let $\otimes_{\mathfrak{h}}^{n} \mathrm{E}$ stand for the complete $n$th tensor power of the Hilbert subspace $E$, endowed with the Hilbertian scalar product and norm, respectively,

$$
\begin{aligned}
\left\langle x_{1} \otimes \cdots \otimes x_{n} \mid \psi_{n}\right\rangle_{\otimes_{\mathfrak{h}}^{n} \mathrm{E}} & =\sum_{j}\left\langle x_{1} \mid y_{1 j}\right\rangle_{\mathrm{E}} \ldots\left\langle x_{n} \mid y_{n j}\right\rangle_{\mathrm{E}}, \\
\left\|\psi_{n}\right\|_{\otimes_{\mathfrak{h}}^{n} \mathrm{E}} & =\left\langle\psi_{n} \mid \psi_{n}\right\rangle_{\otimes_{\mathfrak{h}}^{n} \mathrm{E}}^{1 / 2},
\end{aligned}
$$


where $x_{1} \otimes \cdots \otimes x_{n}, y_{1 j} \otimes \cdots \otimes y_{n j} \in \otimes_{\mathfrak{h}}^{n} \mathrm{E}$ with $x_{t j}, y_{t j} \in \mathrm{E}$ for all $t=1, \ldots, n$ and $\psi_{n}=\sum_{j} y_{1 j} \otimes \cdots \otimes y_{n j}$ denotes a finite sum. Put $\otimes_{\mathfrak{h}}^{0} E=\mathbb{C}$. We use the following short denotation:

$$
x^{\otimes n}=x \otimes \cdots \otimes x, \quad x \in \mathrm{E} .
$$

Replacing the space $E$ by the subspace $\mathbb{C}^{m}$, we similarly define the tensor product $\otimes_{\mathfrak{h}}^{n} \mathbb{C}^{m}$. There is the unitary embed$\operatorname{ding} \otimes_{\mathfrak{h}}^{n} \mathbb{C}^{m} \rightarrow \otimes_{\mathfrak{h}}^{n} \mathrm{E}$. If $m=1$, then $\otimes_{\mathfrak{h}}^{n} \mathbb{C}=\mathbb{C}$.

For any finite sum $\psi_{n}=\sum_{j} y_{1 j} \otimes \cdots \otimes y_{n j}$ from the space $\otimes_{\mathfrak{h}}^{n} \mathbb{C}^{m}$ (or $\otimes_{\mathfrak{h}}^{n} \mathrm{E}$ ), we can to define the finite type $n$-homogeneous Hilbert-Schmidt polynomials:

$$
\mathbb{C}^{m} \ni x \longmapsto\left\langle x^{\otimes n} \mid \psi_{n}\right\rangle_{\otimes_{\mathfrak{h}}^{n} \mathbb{C}^{m}}=\sum_{j} \prod_{t=1}^{n}\left\langle x \mid y_{t j}\right\rangle_{\mathbb{C}^{m}} .
$$

Consider the canonical orthonormal bases:

$$
\begin{gathered}
\mathscr{E}\left(\mathbb{C}^{m}\right)=\left\{\mathfrak{e}_{m 1}, \ldots, \mathfrak{e}_{m m}\right\} \quad \text { in } \mathbb{C}^{m}, \\
\mathscr{E}(\mathrm{E})=\bigcup\left\{\mathscr{E}\left(\mathbb{C}^{m}\right): m \in \mathbb{N}\right\} \quad \text { in } \mathrm{E},
\end{gathered}
$$

where $e_{m l}=\underbrace{(\overbrace{0, \ldots, 0,1}^{l}, 0, \ldots, 0)}$.

If $\mathfrak{g}:\{1, \ldots, n\} \mapsto\{\mathfrak{g}(1), \ldots, \mathfrak{g}(n)\}$ runs over all $n$ elements permutations $\mathfrak{S}(n)$, then the symmetric $n$th tensor power $\odot_{\mathfrak{h}}^{n} \mathbb{C}^{m}$ is defined to be a codomain of the symmetrization mapping:

$$
\begin{gathered}
\otimes_{\mathfrak{h}}^{n} \mathbb{C}^{m} \ni x_{1} \otimes \cdots \otimes x_{n} \longmapsto x_{1} \odot \cdots \odot x_{n}, \\
x_{1} \odot \cdots \odot x_{n}:=\frac{1}{n !} \sum_{\mathfrak{s} \in \mathfrak{S}(n)} x_{\mathfrak{\xi}(1)} \otimes \cdots \otimes x_{\mathfrak{S}(n)},
\end{gathered}
$$

which is an orthogonal projector. Similarly, the symmetric $n$th tensor power $\odot_{\mathfrak{h}}^{n} \mathrm{E}$ can be defined. Clearly, $\odot_{\mathfrak{h}}^{n} \mathbb{C}^{m}$ is a closed subspace in $\odot_{\mathfrak{h}}^{n} \mathrm{E}$.

Given a pair of numbers $(m, n) \in \mathbb{N} \times \mathbb{Z}_{+}$, we consider the $n$-fold tensor power of the canonical mapping $\pi_{m}: \mathfrak{U} \ni$ $u \mapsto \pi_{m}(u) \in U(m)$,

$$
\mathcal{U} \ni u \longmapsto \pi_{m}^{\otimes n}(u) \in \mathscr{L}\left(\odot_{\mathfrak{h}}^{n} \mathbb{C}^{m}\right),
$$

where $\pi_{m}^{\otimes n}(u):=\underbrace{\pi_{m}(u) \otimes \cdots \otimes \pi_{m}(u)}$. If $n=0$, we put $\pi_{m}^{\otimes 0}(u)=1$ for all $u \in \mathcal{U}$ and $m \in \mathbb{N}$. The mapping (36) is Borel and has a continuous restriction to $\mathfrak{U}^{\prime}$, because $\pi_{m}$ has the same property (see Section 2).

Let $\mathfrak{a}_{m} \in \mathbb{C}^{m}$ be an arbitrary fixed element such that $\left\|\mathfrak{a}_{m}\right\|_{\mathbb{C}^{m}}=1$. Then, $\mathfrak{a}_{m}^{\otimes n} \in \odot_{\mathfrak{h}}^{n} \mathbb{C}^{m}$. Using the mapping (36), we can write

$$
\left[\pi_{m}^{\otimes n}(u)\right]\left(\mathfrak{a}_{m}^{\otimes n}\right)=\underbrace{\left[\pi_{m}(u)\right]\left(\mathfrak{a}_{m}\right) \otimes \cdots \otimes\left[\pi_{m}(u)\right]\left(\mathfrak{a}_{m}\right)}_{n} .
$$

To any $n$-homogeneous Hilbert-Schmidt polynomial (33), there corresponds the function

$$
\begin{aligned}
\psi_{n}^{*}(u) & :=\left\langle\left[\pi_{m}^{\otimes n}(u)\right]\left(\mathfrak{a}_{m}^{\otimes n}\right) \mid \psi_{n}\right\rangle_{\otimes_{\mathfrak{h}}^{n} \mathbb{C}^{m}} \\
& =\sum_{j} \prod_{t=1}^{n}\left\langle\left[\pi_{m}(u)\right]\left(\mathfrak{a}_{m}\right) \mid y_{t j}\right\rangle_{\mathbb{C}^{m}}
\end{aligned}
$$

of the variable $u \in \mathcal{U}$. Any cylindrical function of the form $\mathcal{U} \ni u \mapsto\left\langle\left[\pi_{m}(u)\right]\left(\mathfrak{a}_{m}\right) \mid y_{t j}\right\rangle_{\mathbb{C}^{m}}$ has a continuous bounded restriction to $\mathfrak{U}^{\prime}$. Therefore, it is $\chi$-essentially bounded on $\mathfrak{U}$, because $\mathfrak{U} \backslash \mathfrak{U}^{\prime}$ is a $\chi$-negligible set. Consequently, $\psi_{n}^{*} \in L_{\chi}^{\infty}$ and $\left.\psi_{n}^{*}\right|_{\mathfrak{U}^{\prime}}$ is continuous and bounded.

Definition 3. We define $\mathscr{P}_{\mathfrak{h}}^{n}\left(\mathbb{C}^{m}\right)$ to be the space of all functions $\psi_{n}^{*}$ of the variable $u \in \mathfrak{U}$, determined by the finite type n-homogeneous Hilbert-Schmidt polynomials (33).

Lemma 4. For any element $\mathfrak{a}_{m} \in \mathbb{C}^{m}$ such that $\left\|\mathfrak{a}_{m}\right\|_{\mathbb{C}^{m}}=1$ the set

$$
\mathrm{S}^{m}=\left\{x=\left[\pi_{m}(u)\right]\left(\mathfrak{a}_{m}\right): u \in \mathfrak{U}\right\}
$$

coincides with the unit sphere in $\mathbb{C}^{m}$. As a consequence, the one-to-one antilinear corresponding

$$
\odot_{\mathfrak{h}}^{n} \mathbb{C}^{m} \ni \psi_{n} \rightleftarrows \psi_{n}^{*} \in \mathscr{P}_{\mathfrak{h}}^{n}\left(\mathbb{C}^{m}\right)
$$

Holds, and any function $\psi_{n}^{*}$ is independent of the choice of an element $\mathfrak{a}_{m} \in \mathrm{S}^{m}$.

Proof. Suppose, on the contrary, that there is an element $\psi_{n} \in$ $\odot_{\mathfrak{h}}^{n} \mathbb{C}^{m}$ such that $\left\langle x^{\otimes n} \mid \psi_{n}\right\rangle_{\otimes_{\mathfrak{h}}^{n} \mathbb{C}^{m}}=0$ for all $x=\left[\pi_{m}(u)\right]\left(\mathfrak{a}_{m}\right) \epsilon$ $\mathrm{S}^{m}$ with $u \in \mathfrak{U}$. The mapping

$$
\pi_{m}: \mathcal{U} \ni u \longmapsto \pi_{m}(u) \in U(m)
$$

is surjective by surjectivity of the mapping $\pi_{m}$ (see [2, Lemma 3.1]). Hence, the set $S^{m}$ coincides with the unit sphere in $\mathbb{C}^{m}$ and is independent on the choice of an element $\mathfrak{a}_{m}$. By $n$-homogeneity, we have $\left\langle x^{\otimes n} \mid \psi_{n}\right\rangle_{\otimes_{\mathfrak{h}}^{n} \mathbb{C}^{m}}=0$ for all $x \in \mathbb{C}^{m}$.

Apply the following polarization formula for symmetric tensor products (see, e.g., [10, Section 1.5]):

$$
z_{1} \odot \cdots \odot z_{n}=\frac{1}{2^{n} n !} \sum_{1 \leq t \leq n} \sum_{\delta_{t}= \pm 1} \delta_{1} \cdots \delta_{n} x^{\otimes n}
$$

with $x=\sum_{t=1}^{n} \delta_{t} z_{t} \in \mathbb{C}^{m}$, which is valid for all $z_{1}, \ldots, z_{n} \in$ $\mathbb{C}^{m}$. It follows that $\left\langle z_{1} \odot \cdots \odot z_{n} \mid \psi_{n}\right\rangle_{\otimes_{\mathfrak{h}}^{n} \mathbb{C}^{m}}=0$ for all elements $z_{1}, \ldots, z_{n} \in \mathbb{C}^{m}$. Hence, $\psi_{n}=0$, because the subset of all elements $z_{1} \odot \cdots \odot z_{n}$ is total in $\odot_{\mathfrak{h}}^{n} \mathbb{C}^{m}$. As a consequence, the subset

$$
\left\{x^{\otimes n}=\left[\pi_{m}^{\otimes n}(u)\right]\left(\mathfrak{a}_{m}^{\otimes n}\right): u \in \mathfrak{U}\right\}
$$

is also total in $\odot_{\mathfrak{h}}^{n} \mathbb{C}^{m}$. It immediately yields the correspondence (40).

Consider the symmetric Fock space $F$ and its closed subspace $F_{m}$, where

$$
\begin{gathered}
\mathrm{F}:=\mathbb{C} \oplus \mathrm{E} \oplus\left(\odot_{\mathfrak{h}}^{2} \mathrm{E}\right) \oplus\left(\odot_{\mathfrak{h}}^{3} \mathrm{E}\right) \oplus \cdots, \\
\mathrm{F}_{m}:=\mathbb{C} \oplus \mathbb{C}^{m} \oplus\left(\odot_{\mathfrak{h}}^{2} \mathbb{C}^{m}\right) \oplus\left(\odot_{\mathfrak{h}}^{3} \mathbb{C}^{m}\right) \oplus \cdots .
\end{gathered}
$$


We will use the following notations:

$$
\begin{aligned}
(m) & :=(m 1, \ldots, m m), \\
k_{(m)} & :=\left(k_{m 1}, \ldots, k_{m m}\right) \in \mathbb{Z}_{+}^{m}, \\
\left|k_{(m)}\right| & :=k_{m 1}+\cdots+k_{m m}, \\
k_{(m)} ! & :=k_{m 1} ! \cdot \ldots \cdot k_{m m} !
\end{aligned}
$$

As is well known (see, e.g., [11]), the system of symmetric tensor elements, indexed by the set $k_{(m)}$,

$$
\begin{gathered}
\mathscr{E}\left(\odot_{\mathfrak{h}}^{n} \mathbb{C}^{m}\right)=\left\{\boldsymbol{e}_{(m)}^{\otimes k_{(m)}}=\mathfrak{e}_{m 1}^{\otimes k_{m 1}} \odot \cdots \odot \boldsymbol{e}_{m m}^{\otimes k_{m m}}:\right. \\
\left.k_{(m)} \in \mathbb{Z}_{+}^{m} ;\left|k_{(m)}\right|=n\right\}
\end{gathered}
$$

forms an orthogonal basis in the subspace

$$
\odot_{\mathfrak{h}}^{n} \mathbb{C}^{m} \subset \mathrm{F}_{m}
$$

We will also use the following notations:

$$
\begin{gathered}
{[m]:=\{(11),(21,22), \ldots,(m 1, \ldots, m m)\},} \\
\{k\}:=\left\{k_{(1)}, \ldots, k_{(m)}\right\} \in \bigcup_{r=1}^{m} \mathbb{Z}_{+}^{r}, \\
|\{k\}|:=\left|k_{(1)}\right|+\cdots+\left|k_{(m)}\right|, \\
\{k\} !:=k_{(1)} ! \cdot \ldots \cdot k_{(m)} ! .
\end{gathered}
$$

Then, the system of symmetric tensor elements with a fixed $n$, indexed by the sets $[m]$ and $\{k\}$,

$$
\begin{gathered}
\mathscr{E}_{n}=\bigcup_{m \in \mathbb{N}}\left\{\boldsymbol{e}_{[m]}^{\otimes\{k\}}=\mathfrak{e}_{(1)}^{\otimes k_{(1)}} \odot \cdots \odot \boldsymbol{e}_{(m)}^{\otimes k_{(m)}}:\right. \\
\boldsymbol{e}_{(1)}^{\otimes k_{(1)}} \in \mathscr{E}\left(\odot_{\mathfrak{h}}^{\left|k_{(1)}\right|} \mathbb{C}\right), \ldots, \mathfrak{e}_{(m)}^{\otimes k_{(m)}} \in \mathscr{E}\left(\odot_{\mathfrak{h}}^{\left|k_{(m)}\right|} \mathbb{C}^{m}\right) \\
\text { with fixed }|\{k\}|=n\},
\end{gathered}
$$

forms an orthogonal basis in the subspace $\odot_{\mathfrak{h}}^{n} \mathrm{E} \subset \mathrm{F}$. Thus, the system

$$
\mathscr{E}=\left\{\mathscr{E}_{n}: n \in \mathbb{Z}_{+}\right\}
$$

forms an orthogonal basis in the symmetric Fock space $F$.

By virtue of the one-to-one mapping (40), the system of symmetric tensor elements $\mathscr{E}\left(\odot_{\mathfrak{h}}^{n} \mathbb{C}^{m}\right)$ uniquely defines the following corresponding system:

$$
\mathscr{E}_{m, n}^{*} \subset \mathscr{P}_{\mathfrak{h}}^{n}\left(\mathbb{C}^{m}\right),
$$

of the following $\chi_{m}$-integrable cylindrical functions:

$$
\begin{aligned}
\mathbf{e}_{(m)}^{* k_{(m)}}(u):= & \left\langle\left[\pi_{m}^{\otimes n}(u)\right]\left(\mathbf{e}_{m 1}^{\otimes n}\right) \mid \mathfrak{e}_{(m)}^{\otimes k_{(m)}}\right\rangle_{\otimes_{\mathfrak{h}}^{n} \mathbb{C}^{m}} \\
& =\prod_{r=1}^{m}\left\langle\left(\pi_{m} \circ u\right)\left(\mathfrak{e}_{m 1}\right) \mid \mathfrak{e}_{m r}\right\rangle_{\mathbb{C}^{m}}^{k_{m}}
\end{aligned}
$$

of the variable $u \in \mathfrak{U}$, where we take $\mathfrak{a}_{m}=\mathfrak{e}_{m 1}$. Consider the system of functions of the variable $u \in \mathfrak{U}$,

$$
\begin{gathered}
\mathscr{E}_{n}^{*}=\bigcup_{m \in \mathbb{N}}\left\{\mathbf{e}_{[m]}^{*\{k\}}=\mathbf{e}_{(1)}^{* k_{(1)}} \cdots \cdots \mathbf{e}_{(m)}^{* k_{(m)}}:\right. \\
\mathbf{e}_{(1)}^{* k_{(1)}} \in \mathscr{E}_{1, \mid k_{(1)}}^{*}, \ldots, \mathbf{e}_{(m)}^{* k_{(m)}} \in \mathscr{E}_{m,\left|k_{(m)}\right|}^{*} \\
\text { with fixed }|\{k\}|=n\},
\end{gathered}
$$

generated by the system of symmetric tensor elements $\mathscr{E}_{n}$. All these functions belong to the space $\mathscr{L}_{\chi}^{\infty}$ by their definition. Denote

$$
\mathscr{E}^{*}=\left\{\mathscr{E}_{n}^{*}: n \in \mathbb{Z}_{+}\right\}, \quad \mathscr{E}_{m}^{*}=\left\{\mathscr{E}_{m, n}^{*}: n \in \mathbb{Z}_{+}\right\} .
$$

\section{The Hardy-Type Space}

Let $L_{\chi}^{2}$ be the space of square $\chi$-integrable complex functions,$f$ on the space of virtual matrices $\mathcal{U}$. Since $\chi$ is a probability measure, the embedding $\mathscr{L}_{\chi}^{\infty} \subset L_{\chi}^{2}$ holds and

$$
\|f\|_{L_{\chi}^{2}} \leq \operatorname{ess}_{u \in \mathcal{U}}|f(u)|, \quad f \in \mathscr{L}_{\chi}^{\infty} .
$$

Denote by $\mathscr{H}_{\chi_{m}}^{2}$ the $L_{\chi}^{2}$-closure of complex linear spans of the subsystem $\mathscr{E}_{m}^{*}$. As is well known (see, e.g., [12, Theorem 5.6.8]), the space $\mathscr{H}_{\chi_{m}}^{2}$ is isomorphic to the classic Hardy space $\mathscr{H}_{\chi_{m}}^{2}\left(\mathrm{~B}^{m}\right)$ of analytic complex functions on the open unit ball $\mathrm{B}^{m}=\left\{x_{m} \in \mathbb{C}^{m}:\left\|x_{m}\right\|_{\mathbb{C}^{m}}<1\right\}$. Therefore, the following more general definition seems natural (see, also [8]).

Definition 5. The Hardy-type space $\mathscr{H}_{\chi}^{2}$ on the space of virtual unitary matrices $\mathfrak{U}$ is defined to be the $L_{\chi}^{2}$-closure of the complex linear span of the system $\mathscr{E}^{*}$.

Theorem 6. The system $\mathscr{E}^{*}$ of all functions $\mathfrak{e}_{[m]}^{*\{k\}}=$ $\mathbf{e}_{(1)}^{* k_{(1)}} \cdots \mathbf{e}_{(m)}^{* k_{(m)}}$ with $m \in \mathbb{N}$, such that $\mathbf{e}_{(r)}^{* k_{(r)}} \in \mathscr{E}_{r,\left|k_{(r)}\right|}^{*}$ as $r=1, \ldots, m$, forms an orthogonal basis in the Hardy-type spaces $\mathscr{H}_{\chi}^{2}$ with norms

$$
\left\|\mathfrak{e}_{[m]}^{*\{k\}}\right\|_{L_{\chi}^{2}}=\left(\prod_{r=1}^{m} \frac{(r-1) !(k)_{r} !}{\left(r-1+\left|(k)_{r}\right|\right) !}\right)^{1 / 2}
$$

Proof. If $|\{k\}| \neq|\{q\}|$, then from (28), it follows that

$$
\begin{aligned}
\int_{\mathfrak{U}} \mathfrak{e}_{[m]}^{*\{k\}} \cdot \overline{\mathfrak{e}}_{[n]}^{*\{q\}} d \chi \\
\quad=\int_{\mathfrak{U}} \boldsymbol{e}_{[m]}^{*\{k\}}(\exp (\mathfrak{i} \vartheta) u) \cdot \overline{\mathfrak{e}}_{[n]}^{*\{q\}}(\exp (\mathfrak{i} \vartheta) u) d \chi(u) \\
\quad=\frac{1}{2 \pi} \int_{\mathfrak{U}} \mathfrak{e}_{[m]}^{*\{k\}} \overline{\mathbf{e}}_{[n]}^{*\{q\}} d \chi \int_{-\pi}^{\pi} \exp (\mathfrak{i}(|\{k\}|-|\{q\}|) \vartheta) d \vartheta \\
=0 .
\end{aligned}
$$

So, $\mathfrak{e}_{[m]}^{*\{k\}} \perp \mathfrak{e}_{[n]}^{*\{q\}}$ in the space $L_{\chi}^{2}$ if $|\{k\}| \neq|\{q\}|$ for all indices $[m],[n]$. 
Let $|\{k\}|=|\{q\}|$ and $m>n$ for definiteness. If the elements $\mathbf{e}_{[m]}^{*\{k\}}$ and $\mathbf{e}_{[n]}^{*\{q\}}$ are different, then there exists a subindex $m s \in\{11,21,22, \ldots, m 1, \ldots, m m\}$ in the blockindex $[m]=[(11),(21,22), \ldots,(m 1, \ldots, m m)]$ such that $m s \notin$ $\{11,21,22, \ldots, n 1, \ldots, n n\}$, where $[n]=[(11),(21,22), \ldots$, $(n 1, \ldots, n n)]$. The formula (28) implies that for the group of shifts $Q_{g_{m s}(9)}$ generated by elements $g_{m s}(\vartheta) \in U_{s}^{2}(m)$ with the subindex $m s$,

$$
\begin{aligned}
\int_{\mathfrak{U}} \mathbf{e}_{[m]}^{*\{k\}} \cdot \overline{\mathbf{e}}_{[n]}^{*\{q\}} d \chi \\
\quad=\int_{\mathfrak{U}} Q_{g_{m s}(\vartheta)} \mathbf{e}_{[m]}^{*\{k\}} \cdot Q_{g_{m s}(\vartheta)} \overline{\mathbf{e}}_{[n]}^{*\{q\}} d \chi \\
\quad=\frac{1}{2 \pi} \int_{\mathfrak{U}} \mathbf{e}_{[m]}^{*\{k\}} \cdot \overline{\mathbf{e}}_{[n]}^{*\{q\}} d \chi \int_{-\pi}^{\pi} \exp \left(\mathfrak{i} k_{m s} \vartheta\right) d \vartheta=0 .
\end{aligned}
$$

Hence, $\mathbf{e}_{[m]}^{*\{k\}} \perp \mathbf{e}_{[n]}^{*\{q\}}$ in $L_{\chi}^{2}$.

Let now $|\{k\}|=|\{q\}|$ and $m=n$. If $\mathbf{e}_{[m]}^{*\{k\}} \neq \mathbf{e}_{[n]}^{*\{q\}}$, then $\{k\} \neq\{q\}$. Hence, there exists a sub-index $r s$ in the blockindex $[m]=[n]$ such that $k_{r s} \neq q_{r s}$. Similarly as previous mentioned, applying the formula (28) to the group of shifts $Q_{g_{r s}(9)}$ generated by elements $g_{r s}(9) \in U_{s}^{2}(r)$ with the subindex $r s$, we get

$$
\begin{aligned}
\int_{\mathfrak{U}} \mathfrak{e}_{[m]}^{*\{k\}} \cdot \overline{\mathfrak{e}}_{[n]}^{*\{q\}} d \chi \\
\quad=\frac{1}{2 \pi} \int_{\mathfrak{U}} \mathfrak{e}_{[m]}^{*\{k\}} \overline{\mathfrak{e}}_{[n]}^{*\{q\}} d \chi \int_{-\pi}^{\pi} \exp \left(\mathfrak{i}\left(k_{r s}-q_{r s}\right) \vartheta\right) d \mathcal{V} \\
\quad=0 .
\end{aligned}
$$

Hence, in this case also $\mathbf{e}_{[m]}^{*\{k\}} \perp \mathbf{e}_{[n]}^{*\{q\}}$ under the measure $\chi$.

Let $g_{r}=\left(\mathbb{1}_{r}, w_{r}\right) \in U^{2}(r)$ and $u \in \mathcal{U}$. Using (11) and (52), we have

$$
\begin{aligned}
\int_{U^{2}(r)} & Q_{g_{r}}\left|\mathbf{e}_{(r)}^{*(k)_{r}}\right|^{2}(u) d\left(\chi_{r} \otimes \chi_{r}\right)\left(g_{r}\right) \\
= & \left.\int_{U(r)} \prod_{l=1}^{r}\left|\left\langle\left[w_{r}^{-1} \pi_{r}(u)\right]\left(\mathbf{e}_{r 1}\right) \mid \mathbf{e}_{r l}\right\rangle_{\mathbb{C}^{r}}\right|^{k_{r l}}\right|^{2} d \chi_{r}\left(w_{r}\right) .
\end{aligned}
$$

However, the previous integral with the Haar measure $\chi_{r}$ is independent of $\pi_{r}(u) \in U(r)$. It follows that

$$
\begin{aligned}
\int_{U^{2}(r)} & Q_{g_{r}}\left|\mathbf{e}_{(r)}^{*(k)_{r}}\right|^{2}(u) d\left(\chi_{r} \otimes \chi_{r}\right)\left(g_{r}\right) \\
= & \int_{U(r)} \prod_{l=1}^{r}\left|\left\langle w_{r}^{-1}\left(\mathfrak{e}_{r 1}\right) \mid \mathfrak{e}_{r l}\right\rangle_{\mathbb{C}^{r}}^{k_{r l}}\right|^{2} d \chi_{r}\left(w_{r}\right) \\
= & \frac{(r-1) !(k)_{r} !}{\left(r-1+\left|(k)_{r}\right|\right) !}=\left\|\mathbf{e}_{(r)}^{*(k)_{r}}\right\|_{L_{\chi_{r}}^{2}}^{2}
\end{aligned}
$$

by the well-known formula [12, Section 1.4.9]. Using the formula (27) $m$-times for $r=1, \ldots, m$, we get

$$
\begin{aligned}
\int_{\mathfrak{U}}\left|\mathbf{e}_{[m]}^{*\{k\}}\right|^{2} d \chi & \\
& =\int_{\mathfrak{U}} d \chi(u) \prod_{r=1}^{m} \int_{U^{2}(r)} Q_{g_{r}}\left|\mathbf{e}_{(r)}^{*(k)}\right|^{2}(u) d\left(\chi_{r} \otimes \chi_{r}\right)\left(g_{r}\right) \\
& =\prod_{r=1}^{m}\left\|\mathfrak{e}_{(r)}^{* k_{(r)}}\right\|_{L_{\chi_{r}}^{2}}^{2}
\end{aligned}
$$

because $\int_{\mathfrak{L}} d \chi=1$. It follows that

$$
\left\|\boldsymbol{e}_{[m]}^{*\{k\}}\right\|_{L_{\chi}^{2}}^{2}=\prod_{r=1}^{m}\left\|\boldsymbol{e}_{(r)}^{* k_{(r)}}\right\|_{L_{\chi_{r}}^{2}}^{2}=\prod_{r=1}^{m} \frac{(r-1) !(k)_{r} !}{\left(r-1+\left|(k)_{r}\right|\right) !}
$$

for all $\mathbf{e}_{[m]}^{*\{k\}}=\mathbf{e}_{(1)}^{* k_{(m)}} \cdots \mathbf{e}_{(m)}^{* k_{(m)}}$.

As is known (see, e.g., [11]), the system $\mathscr{E}_{m}$ of symmetric tensors $\mathbf{e}_{(m)}^{\otimes(k)_{m}}$ with a fixed $m$ forms an orthogonal basis in the symmetric Fock space $F_{m}$ with norms $\left\|\mathbf{e}_{(m)}^{\otimes(k)_{m}}\right\|_{F_{m}}=$ $\sqrt{(k)_{m} ! /\left|(k)_{m}\right| !}$. Similarly, the system $\mathscr{E}$ of symmetric tensors $\mathbf{e}_{[m]}^{\otimes\{k\}}=\mathbf{e}_{(1)}^{\otimes(k)_{1}} \odot \cdots \odot \mathbf{e}_{(m)}^{\otimes k_{(m)}}$ with all $m \in \mathbb{N}$, such that $\mathbf{e}_{(r)}^{\otimes(k)_{r}} \in$ $\mathscr{E}_{r,\left|(k)_{r}\right|}$ as $r=1, \ldots, m$, forms an orthogonal basis in the symmetric Fock space $\mathrm{F}$ with norms $\left\|\boldsymbol{e}_{[m]}^{\otimes\{k\}}\right\|_{\mathrm{F}}=\sqrt{\{k\} ! /|\{k\}| !}$.

Combining Lemma 4, Theorem 6, and [12, Theorem 5.6.8], we obtain the following.

Theorem 7. Antilinear extensions of the one-to-one mappings between the orthonormal bases

$$
\begin{aligned}
\frac{\mathbf{e}_{(m)}^{\otimes(k)_{m}}}{\left\|\mathbf{e}_{(m)}^{\otimes(k)_{m}}\right\|_{F_{m}}} & \rightleftarrows \frac{\mathbf{e}_{(m)}^{*(k)_{m}}}{\left\|\mathbf{e}_{(m)}^{*(k)_{m}}\right\|_{L_{\chi m}^{2}}}, \\
\frac{\mathbf{e}_{[m]}^{\otimes\{k\}}}{\left\|\mathbf{e}_{[m]}^{\otimes\{k\}}\right\|_{\boldsymbol{F}}} & \rightleftarrows \frac{\mathbf{e}_{[m]}^{*\{k\}}}{\left\|\mathfrak{e}_{[m]}^{*\{k\}}\right\|_{L_{\chi}^{2}}},
\end{aligned}
$$

uniquely define the corresponding anti-linear isometric isomorphisms

$$
\mathrm{F}_{m} \simeq \mathscr{H}_{\chi_{m}}^{2}\left(\mathrm{~B}^{m}\right), \quad \mathrm{F} \simeq \mathscr{H}_{\chi}^{2} .
$$

Reasoning by analogy with [8, Proposition 6.1 and Theorem 7.1], it is easy to show that the Hardy space $\mathscr{H}_{\chi}^{2}$ possesses the reproducing kernel of a Cauchy type

$$
\begin{aligned}
\mathfrak{S}(v, u) & =\sum_{n \in \mathbb{Z}_{+}} \sum_{\{\{k\} \mid=n} \frac{\mathfrak{e}_{[m]}^{*\{k\}}(v) \overline{\mathfrak{e}}_{[m]}^{*\{k\}}(u)}{\left\|\mathfrak{e}_{[m]}^{*\{k\}}\right\|_{L_{\chi}^{2}}^{2}} \\
& =\prod_{m=1}^{\infty}\left(1-\left\langle\left(\pi_{m} \circ v\right)\left(\mathfrak{e}_{m 1}\right) \mid\left(\pi_{m} \circ u\right)\left(\mathbf{e}_{m 1}\right)\right\rangle_{\mathrm{E}}\right)^{-m},
\end{aligned}
$$


with $u, v \in \mathcal{U}$, where the sum $\sum_{|\{k\}|=n}$ is over all indices $\{k\} \in$ $\left\{X_{r=1}^{m} \mathbb{Z}_{+}^{r}: m \in \mathbb{N}\right\}$ such that $|\{k\}|=n$. As a consequence, the integral representation of any function $f \in \mathscr{H}_{\chi}^{2}$,

$$
f(\lambda v)=\int_{\mathfrak{U}} f(u) \mathfrak{C}(\lambda v, u) d \chi(u)
$$

gives a unique analytic extension in the complex variable $\lambda \epsilon$ $\mathrm{B}^{1}$ for all elements $v \in \mathfrak{U}$ such that

$$
\sum_{m \in \mathbb{N}} m\left\|\left(\pi_{m} \circ v\right)\left(\mathfrak{e}_{m 1}\right)\right\|_{\mathbb{C}^{m}}^{2}<\infty .
$$

\section{References}

[1] Y. A. Neretin, "Hua-type integrals over unitary groups and over projective limits of unitary groups," Duke Mathematical Journal, vol. 114, no. 2, pp. 239-266, 2002.

[2] G. Olshanski, "The problem of harmonic analysis on the infinite-dimensional unitary group," Journal of Functional Analysis, vol. 205, no. 2, pp. 464-524, 2003.

[3] D. Pickrell, "Measures on infinite dimensional Grassmann manifolds," Journal of Functional Analysis, vol. 70, no. 2, pp. 323-356, 1987.

[4] S. Kerov, G. Olshanski, and A. Vershik, "Harmonic analysis on the infinite symmetric group: a deformation of regular representation," Comptes Rendus de l'Académie des Sciences. Series I, vol. 316, pp. 773-778, 1993.

[5] A. Borodin and G. Olshanski, "Harmonic analysis on the infinite-dimensional unitary group and determinantal point processes," Annals of Mathematics, vol. 161, no. 3, pp. 1319-1422, 2005.

[6] E. Tomas, “On Prohorov's criterion for projective limits," Operator Theory, vol. 168, pp. 251-261, 2006.

[7] Y. Yamasaki, "Projective limit of Haar measures on $\mathrm{O}(\mathrm{n})$," Research Institute for Mathematical Sciences, vol. 8, pp. 141-149, 1972/73.

[8] O. Lopushansky and A. Zagorodnyuk, "Hardy type spaces associated with compact unitary groups," Nonlinear Analysis: Theory, Methods and Applications, vol. 74, no. 2, pp. 556-572, 2011.

[9] N. Bourbaki, Integration II, Springer, Berlin, Germany, 2004.

[10] K. Floret, "Natural norms on symmetric tensor products of normed spaces," Note di Matematica, vol. 17, pp. 153-188, 1997.

[11] M. Reed and B. Simon, Methods of Modern Mathematical Physics, Volume II, Academic Press, New York, NY, USA, 1975.

[12] W. Rudin, Function Theory in the Unit Ball of $\mathbb{C}^{n}$, Springer, Berlin, Germany, 1980. 


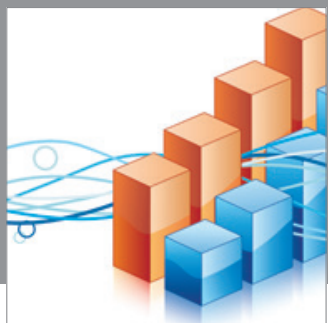

Advances in

Operations Research

mansans

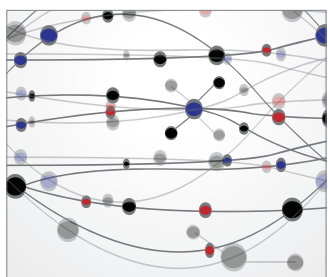

The Scientific World Journal
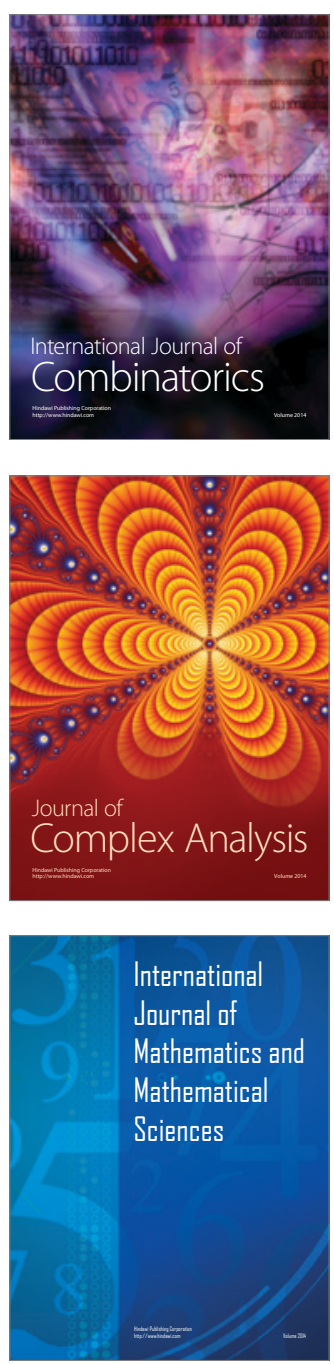
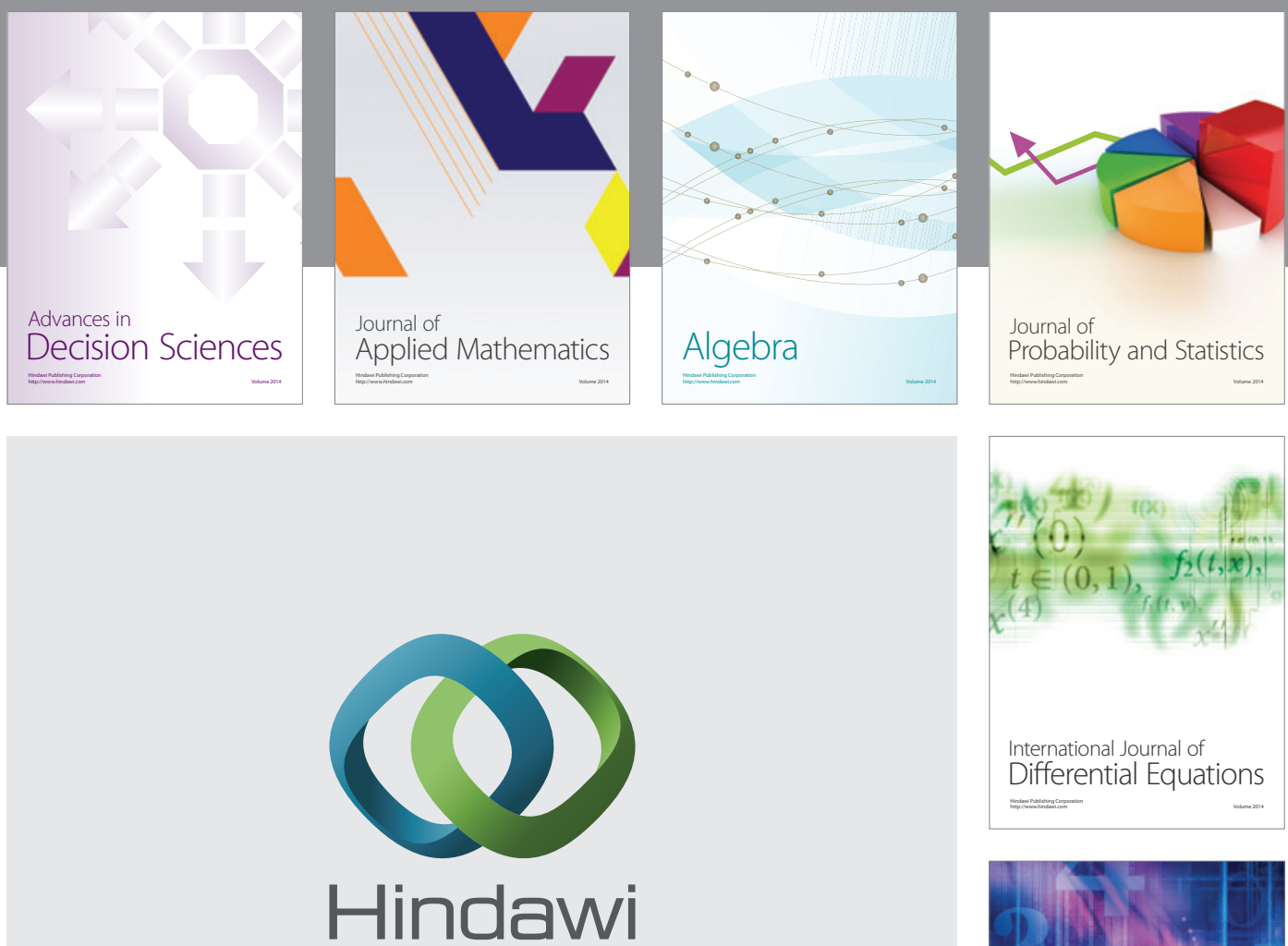

Submit your manuscripts at http://www.hindawi.com
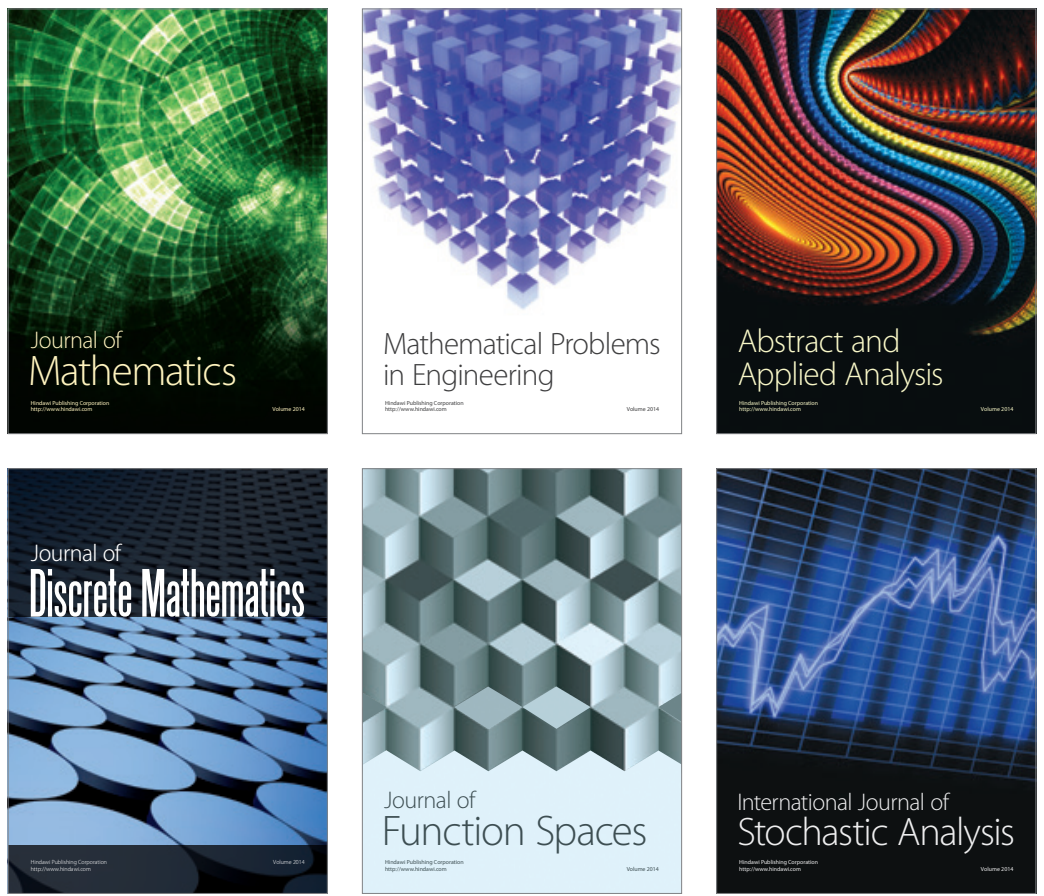

Journal of

Function Spaces

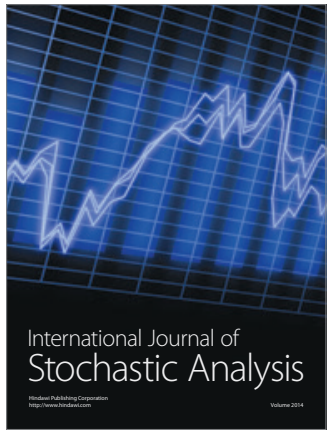

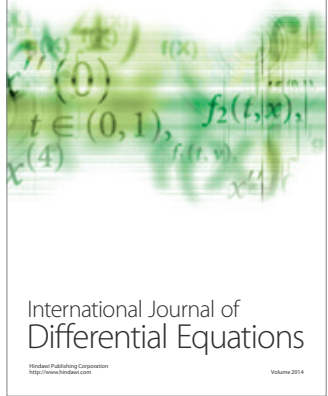
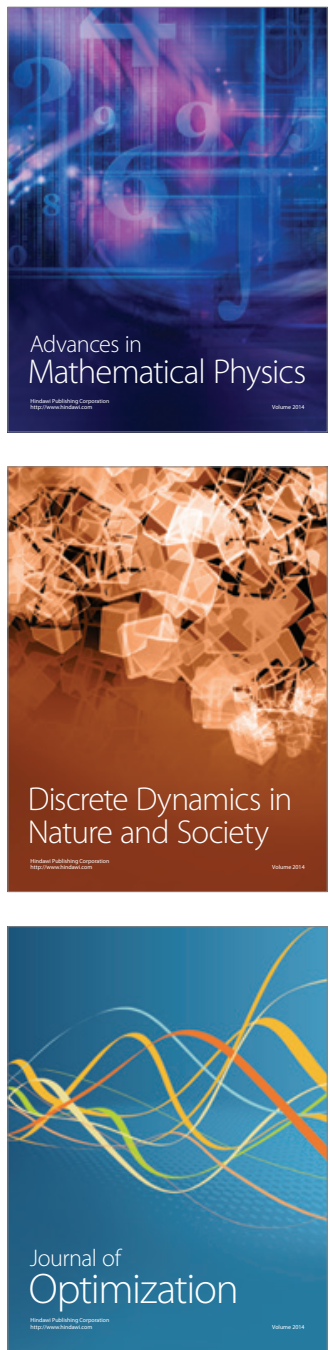